\title{
The course of fatigue and its correlates in colorectal cancer survivors: a prospective cohort study of the PROFILES registry
}

\author{
Olga Husson $^{1,2}$ • Floortje Mols ${ }^{1,2}$ - Lonneke V. van de Poll-Franse ${ }^{1,2}$. \\ Melissa S. Y. Thong ${ }^{1,2}$
}

Received: 15 January 2015 / Accepted: 8 June 2015 / Published online: 28 June 2015

(C) The Author(s) 2015. This article is published with open access at Springerlink.com

\begin{abstract}
Purpose Colorectal cancer (CRC) survivors who remain fatigued during long-term follow-up are at risk for worse health outcomes and need relevant interventions most. The aim of this study is to prospectively assess cancer-related fatigue (CRF) and four categories of CRF correlates (clinical characteristics, demographic characteristics, behavior/well-being, functional status).

Methods CRC survivors diagnosed between 2000 and 2009, as registered in the population-based Eindhoven Cancer Registry, completed the Fatigue Assessment Scale at three annual time points. Linear mixed models were used to assess the course of CRF and identify its correlates.

Results CRF levels were relatively stable over time. Being female, young ( $\leq 65$ years of age), and single; having a low educational level; treatment with chemotherapy; and having one or more comorbid conditions were associated with higher CRF scores. Years since diagnosis, radiotherapy, and disease stage were not related to CRF over time.
\end{abstract}

This manuscript will be presented as a paper at the Supportive Care in Cancer MASCC/ISOO 2015 International Symposium in Copenhagen on June 25-27, 2015.

This manuscript has been prepared in accordance with the style of the journal, and all authors have approved of its contents. This manuscript is not being considered for publication elsewhere, and the findings of this manuscript have not been previously published.

Olga Husson

o.husson@tilburguniversity.edu

1 Center of Research on Psychology in Somatic diseases (CoRPS), Department of Medical and Clinical Psychology, Tilburg University, Warandelaan 2, PO Box 90153, 5000 LE Tilburg, The Netherlands

2 Comprehensive Cancer Centre the Netherlands (CCCN), Eindhoven Cancer Registry, Eindhoven, The Netherlands
Significant between- and within-subject effects were found for all well-being factors (social, emotional, and cognitive functioning, and global quality of life), symptoms (anxiety, depression, pain, and insomnia), and functional status (physical and role functioning, physical activity levels) in relation to CRF.

The differences in CRF levels could, for a large part, be attributed to differences in behavior/well-being (59 \%), functional status (37\%), and, to a lesser extent, to sociodemographic $(4 \%)$ and clinical characteristics $(8 \%)$.

Conclusion This study showed that sociodemographic and clinical factors were associated with CRF levels over time among CRC survivors; however, behavior/well-being and functional status explained a larger part of the variance in levels of CRF.

Keywords Behavior · Cancer · Fatigue - Functional status · Survivorship $\cdot$ Well-being

\section{Introduction}

Improvements in early detection and treatment have increased colorectal cancer (CRC) survival rates [1]. In the Netherlands, there were about 77,000 CRC survivors in 2009 , which is expected to increase to 121,000 in 2020 [2]. The majority $(>56 \%$ ) of the CRC patients survive relatively long $(>10$ years after diagnosis) [3]. Despite these advancements, cancer treatments may put cancer survivors at risk for long-term side effects. With more patients surviving longer, the long-term effects of cancer and its treatment on the survivors' wellbeing are of increasing importance.

An important long-term effect of cancer and its treatment is cancer-related fatigue (CRF). CRF is defined as a subjective sense of physical, emotional, and/or cognitive tiredness or 
exhaustion that is not proportional to recent activity and interferes with usual functioning [4]. CRF is the most common symptom experienced by cancer survivors, with prevalence rates of up to $99 \%$ among survivors directly after diagnosis and during treatment [5]. Among CRC survivors, prevalence rates range from $41 \%$ among short-term survivors $(<5$ years since diagnosis) and $35 \%$ among long-term survivors ( $\geq 5$ years since diagnosis) [6]. Cancer survivors indicate that $\mathrm{CRF}$ is more troublesome and has a greater negative impact on health-related quality of life (HRQoL) and daily activities than other distressing cancer symptoms, like pain and depression [7]. Given the high prevalence and impact of CRF, it is an important target for identification and treatment.

The causes underlying CRF are still not well understood. However, it is hypothesized that CRF is rooted in both biology and behavior [8]. In a recent recommendation paper, a conceptual framework was proposed whereby five categories of CRF correlates were identified: clinical characteristics (e.g., disease stage, treatment), demographic characteristics (e.g., age, sex), behavior/well-being (e.g., anxiety, pain), functional status (e.g., performance status), and biologic status (e.g., cytokine function) [8]. Although studies show that prevalence rates of CRF diminish during post-treatment follow-up, more insight is needed into the longitudinal course of CRF during long-term survivorship and the correlates that contribute to CRF over time. Survivors who remain fatigued during longterm follow-up are at risk for worse health outcomes and need relevant interventions most [8]. To achieve personalized management of CRF, insight into the correlates of CRF among long-term survivors is needed to identify subgroups of survivors at risk of remaining fatigued and also the correlates for intervention. Therefore, the aim of this study is to prospectively assess CRF and its correlates among CRC survivors. An adapted version of the conceptual CRF correlates framework will be used for our study (Fig. 1). We have not included the biological status dimension of the original model in our study, as the Patient Reported Outcomes Following Initial treatment and Long term Evaluation of Survivorship (PROFILES) registry currently does not collect biological data.

\section{Methods}

\section{Setting and population}

This study is part of a longitudinal, population-based survey among CRC survivors registered within the Eindhoven Cancer Registry (ECR) of the Comprehensive Cancer Centre Netherlands. The ECR records data on all individuals who are newly diagnosed with cancer in the southern part of the Netherlands, an area with 2.3 million inhabitants, 18 hospital locations, and two large radiotherapy institutes. All individuals diagnosed with CRC between 2000 and 2009 as registered in the ECR were eligible for participation. Survivors who had died prior to start of the study (according to the Central Bureau for Genealogy which collects information on all deceased Dutch citizens via the civil municipal registries and hospital records) or had unverifiable addresses were excluded. Also, survivors with severe cognitive impairment (e.g., dementia) were excluded because it was expected that they would have difficulties completing the questionnaires without assistance. The study started in December 2010 (T1), and respondents received a subsequent questionnaire in 2011 (T2) and 2012 (T3). A complete overview of the

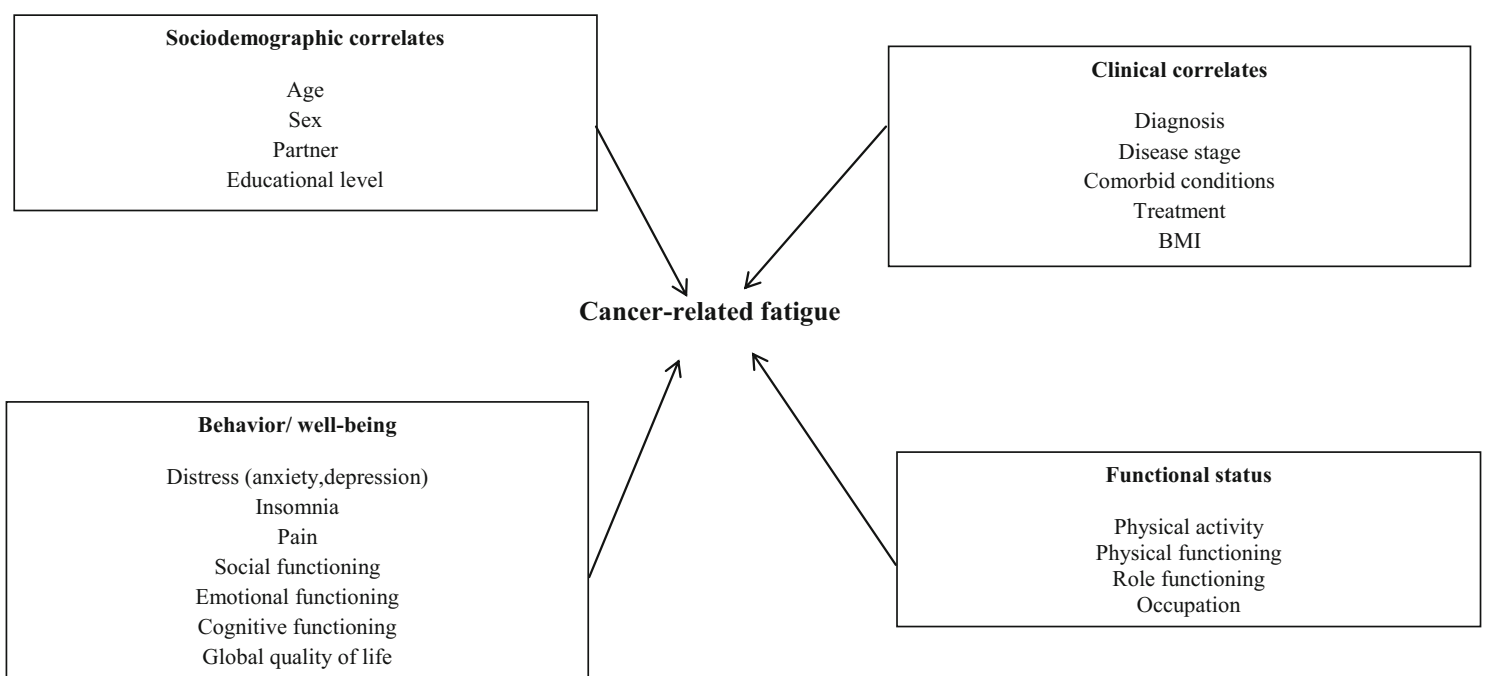

Note: $B M I=$ body mass index

Fig. 1 Correlates of cancer-related fatigue 
selection of survivors can be found in Fig. 2. Ethical approval for the study was obtained from a local certified Medical Ethics Committee of the Maxima Medical Centre Veldhoven, the Netherlands.

\section{Data collection}

Data collection was done within PROFILES. PROFILES is a registry for the study of the physical and psychosocial impact of cancer and its treatment from a dynamic, growing population-based cohort of both short- and long-term cancer survivors. PROFILES contains a large web-based component and is linked directly to clinical data from the ECR. Details of the data collection method were previously described [9].

\section{Study measures}

\section{Fatigue}

CRF was assessed with the Fatigue Assessment Scale (FAS), a questionnaire consisting of ten items. The response scale is a five-point scale ( 1 never to 5 always), and total scores can

Fig. 2 Flow chart of the study

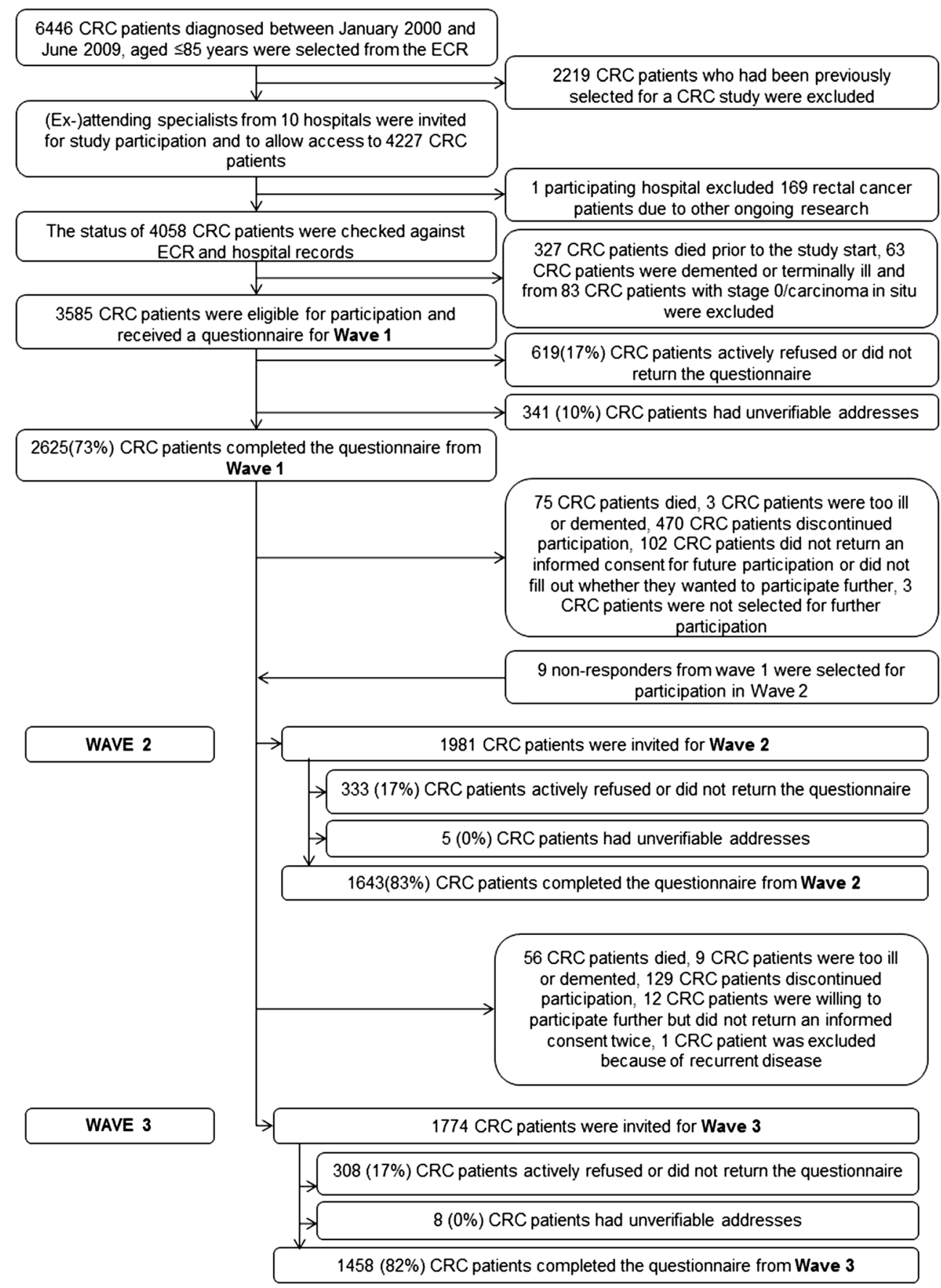


range from 10 to 50 . Survivors can be divided into two groups based on total FAS scores [10]: not fatigued (as defined by a score of 10 to 21 ) and fatigued (22 to 50). The psychometric properties are good [11].

\section{Clinical characteristics}

Clinical information was available from the ECR that routinely collects data on tumor characteristics, including date of diagnosis, tumor stage, primary treatment, and survivors' background characteristics. Comorbidity at the time of survey was assessed with the adapted Self-Administered Comorbidity Questionnaire (SCQ) [12]. Questions on height and weight were added to the questionnaire to calculate body mass index (BMI).

\section{Sociodemographic characteristics}

Questions on marital status and educational level were added to the questionnaire.

\section{Behavior/well-being}

Well-being (or HRQoL) was measured by the Dutch version of the validated European Organization for Research and Treatment of Cancer Quality of Life Questionnaire-Core 30 (EORTC QLQ-C30) [13]. This 30-item HRQoL questionnaire consists of five functional scales, of which only the emotional, social, and cognitive functioning scales were used in this study, a global health status scale, three symptom scales, and single symptom items (only the insomnia and pain items will be used). Answer categories range from one (not at all) to four (very much). All scales are linearly transformed according to the guidelines of the EORTC [13], to reach a scale range of 0 to 100. A higher score on the functional scales and global quality of life implies better HRQoL, while a higher score on the symptom scales and items implies more symptoms.

Symptoms of anxiety and depression were assessed with the Hospital Anxiety and Depression Scale (HADS), with seven items each for assessing both anxiety and depression [14]. All items were scored on a zero- to three-point scale, with higher scores indicating more symptoms. A sum score was calculated for both scales, which can range from 0 to 21 .

\section{Functional status}

Functional status was assessed with the physical and role functioning scales of the EORTC QLQ-C30 [13] and a selfdesigned question on employment status which was added to the questionnaire.

Physical activity level as a dimension of functional status was assessed with questions derived from the validated European Prospective Investigation into Cancer (EPIC) Physical Activity
Questionnaire [15]. Survivors were asked how much time they spend on the following activities (average number of hours per week (h/week), in summer and winter separately): walking, bicycling, gardening, housekeeping, and sports. Six separate sports could be specified. Total PA was calculated by summing hours/week of all activities. To include an estimate of intensity, metabolic equivalent intensity values (MET) were assigned to each activity, according to the compendium of physical activities $[16,17]$. The duration of moderate to vigorous physical activity (MVPA) was assessed as time (h/week) spent on walking, bicycling, gardening, and sports ( $\geq 3$ MET), excluding housekeeping and light intensity sports $(<3$ MET).

\section{Statistical analyses}

Differences in sociodemographic and clinical variables between respondents and non-respondents were examined with analysis of variance (ANOVA) for continuous variables and chi-square tests for categorical variables, where appropriate. Potential non-response bias during follow-up was assessed by comparing the characteristics of survivors who responded to all three waves (full respondent), with those who responded to either two or one data wave. All further analyses were based on survivors who responded to all three waves.

The course of CRF and the individual associations between each independent variable and CRF over time were analyzed using linear mixed models (covariance pattern model with an unstructured error covariance matrix and maximum likelihood estimation) [18]. This technique uses data efficiently by also including incomplete cases in the analyses. As a result of this, bias is limited and statistical power is preserved. Linear mixed models were used to adjust for the dependence of observations. In order to correctly interpret all model parameters, all continuous variables were grand mean centered $[18,19]$. Time was analyzed as a regular categorical predictor with three levels (i.e., three time points). Sociodemographic and clinical variables were analyzed as time-invariant predictors (i.e., baseline characteristics were used).

In the first step, we developed a longitudinal linear mixed model by putting CRF as a dependent variable in the regression equation and one variable of interest, time, and the possible confounders as independent variables. Confounders were chosen based on a priori assumptions: age, gender, having a partner, educational level, years since diagnosis, disease stage, treatment, and number of comorbidities. The confounders were also assessed for their association with CRF.

In the second step of the longitudinal data analyses, we examined the between-subject and within-subject effects for each continuous independent variable separately. Differences in CRF can occur because survivors differ in the independent variable (between-subject) or because a survivor differs over time in the independent variable (within-subject). Therefore, the between-subject estimate determined if differences in the 
independent variable between survivors resulted in differences in CRF. This estimate was represented by survivors' average amount of CRF reported during the study across the three measurements. The within-subject estimate determined causal relations by assessing if changes in the independent variable within a participant were related to changes in CRF and was represented by the difference between survivors' CRF at a certain point in time and his/her average CRF during the study. The between-subject and within-subject estimates were simultaneously entered in the linear mixed models together with the possible confounders and two dummies for time, with T1 as reference category, as independent variables and $\mathrm{CRF}$ as dependent variable.

Furthermore, cross-sectional multivariate linear regression analyses were used to assess the conjoint association between multiple independent variables and CRF at T1. The explained variance at $\mathrm{T} 1$ was assessed for the following domains: sociodemographic factors, clinical factors, behavior/well-being, and functional status. Linear regression analyses were more appropriate than linear mixed models to assess the explained variance, and therefore, we examined the conjoint association at one time point instead of over time.

Analyses were performed in IBM SPSS 22.0, using significance level of $\alpha=0.05$.

\section{Results}

\section{Sociodemographic and clinical characteristics of respondents and non-respondents at $\mathrm{T} 1$}

The questionnaire was completed by $73 \%$ of survivors $(n=$ $2625)$ at T1, $83 \%(n=1643)$ at T2, and $82 \%(n=1458)$ at T3. Respondents at T1 were significantly younger, were more often male, had a longer time since diagnosis, were more often diagnosed with stage I disease, and were more often treated with radiotherapy compared to non-respondents at T1 (all $p<0.05$; data not shown). Furthermore, respondents at $\mathrm{T} 1$ more often received radiotherapy and were more often male compared to survivors with unverifiable addresses at $\mathrm{T} 1$ $(p<0.05$; data not shown).

\section{Differences between CRC survivors who completed one or more than one questionnaire}

CRC survivors who completed only one questionnaire were older at time of first enrollment, were more often female, were less likely to have a partner, were less likely to have a job, were not meeting the physical activity guidelines, and were more fatigued compared to those who completed two or more questionnaires. In addition, they were more often diagnosed with disease stage IV and, thus, less likely to receive radiotherapy and surgery as primary treatment.
CRC survivors who completed only one or two questionnaires had a lower educational level and reported more comorbid conditions, more symptoms (anxiety, depression, pain), less functioning (physical, role, emotional, cognitive, and social), and lower quality of life compared to those who completed all three questionnaires.

No differences were found in time since diagnosis, receiving chemotherapy in addition to surgery as primary treatment, BMI, and insomnia (Table 1).

\section{Factors longitudinally associated with CRF}

Figure 3 shows that the CRF levels were relatively stable over time. Mixed model analysis also showed no significant effect of time (Table 2).

All sociodemographic factors were significantly associated with CRF levels over time (Table 2). Male CRC survivors reported, on average, 1.24 points lower levels of CRF than female survivors. Furthermore, survivors $\leq 65$ years of age reported, on average, 0.62 points higher CRF levels compared to survivors $>65$ years of age. CRC survivors who have a partner scored 0.82 points lower on $\mathrm{CRF}$ than survivors without a partner, and survivors with a high educational level score 1 point lower on $\mathrm{CRF}$ compared to those with a medium or low educational level.

Regarding clinical characteristics, survivors who received chemotherapy as primary treatment scored 0.86 points higher on CRF compared to their counterparts. Survivors with two or more comorbid conditions reported higher levels of CRF compared to those with one ( 0.80 points) and no comorbid conditions (1.63 points). BMI was positively associated with levels of CRF $(B=0.12)$. Years since diagnosis, disease stage, and radiotherapy as primary treatment were not associated with levels of CRF over time.

Significant between- and within-subject effects were found for all well-being (social, emotional, cognitive functioning, and global quality of life) and symptom factors (anxiety, depression, pain, and insomnia).

In addition, significant between- and within-subject effects were found for all functional status variables (physical and role functioning, MVPA levels), except for occupational status which was not related to CRF levels over time among CRC survivors (Table 2).

\section{Conjoint associations with CRF}

At T1, $4 \%$ of the differences in levels of CRF could be explained by sociodemographic factors, $8 \%$ by clinical factors, $59 \%$ by behavior/well-being, and $37 \%$ by functional status. Of the behavior/well-being correlates, the highest variance in CRF could be attributed to differences in levels of depression (14\%), cognitive functioning (15\%), and global quality of life $(12 \%)$. Of the functional status correlates, the highest variance in CRF could be explained by physical 
Table 1 Sociodemographic and clinical characteristics of CRC survivors at T1

\begin{tabular}{|c|c|c|c|c|}
\hline & One wave $N=891$ & Two waves $N=281$ & Full response (three waves) $N=1453$ & $p$ value \\
\hline \multicolumn{5}{|l|}{ Sociodemographic characteristics } \\
\hline Sex & & & & 0.02 \\
\hline Male & $458(51 \%)$ & $157(56 \%)$ & $833(57 \%)$ & \\
\hline Female & $433(49 \%)$ & $124(44 \%)$ & $620(43 \%)$ & \\
\hline Age mean (SD) & $71.3(9.4)$ & $69.4(9.9)$ & $68.2(9.3)$ & $<0.001$ \\
\hline Age (years) & & & & $<0.001$ \\
\hline$\leq 65$ & $234(26 \%)$ & $85(30 \%)$ & $536(37 \%)$ & \\
\hline$>65$ & $657(74 \%)$ & $196(70 \%)$ & $917(63 \%)$ & \\
\hline \multicolumn{5}{|l|}{ Partner } \\
\hline Yes & $626(71 \%)$ & $222(80 \%)$ & $1136(79 \%)$ & $<0.001$ \\
\hline Educational level & & & & $<0.001$ \\
\hline Low & $235(27 \%)$ & $67(24 \%)$ & $218(15 \%)$ & \\
\hline Middle & $510(59 \%)$ & $159(58 \%)$ & $899(62 \%)$ & \\
\hline High & $125(14 \%)$ & $50(18 \%)$ & $333(23 \%)$ & \\
\hline \multicolumn{5}{|l|}{ Clinical characteristics } \\
\hline Years since diagnosis mean (SD) & $5.3(2.8)$ & $5.1(2.8)$ & $5.1(2.8)$ & 0.46 \\
\hline Years since diagnosis & & & & 0.55 \\
\hline$\leq 5$ & $514(58 \%)$ & $170(61 \%)$ & $828(57 \%)$ & \\
\hline$>5$ & $377(42 \%)$ & $111(39 \%)$ & $625(43 \%)$ & \\
\hline Stage & & & & $<0.001$ \\
\hline I & $262(30 \%)$ & $77(28 \%)$ & $441(31 \%)$ & \\
\hline II & $335(38 \%)$ & $101(36 \%)$ & $511(36 \%)$ & \\
\hline III & $220(25 \%)$ & $86(31 \%)$ & $418(30 \%)$ & \\
\hline IV & $59(7 \%)$ & $14(5 \%)$ & $41(3 \%)$ & \\
\hline \multicolumn{5}{|l|}{ Chemotherapy } \\
\hline Yes & $255(29 \%)$ & $88(31 \%)$ & $428(30 \%)$ & 0.68 \\
\hline \multicolumn{5}{|l|}{ Radiotherapy } \\
\hline Yes & $243(27 \%)$ & $85(30 \%)$ & $474(33 \%)$ & 0.02 \\
\hline \multicolumn{5}{|l|}{ Surgery } \\
\hline Yes & $872(98 \%)$ & $279(99 \%)$ & $1447(99 \%)$ & $<0.001$ \\
\hline Number of comorbid conditions & & & & 0.01 \\
\hline None & $192(24 \%)$ & $61(23 \%)$ & $361(26 \%)$ & \\
\hline One & $208(26 \%)$ & $69(26 \%)$ & $432(31 \%)$ & \\
\hline Two or more & $390(49 \%)$ & $139(52 \%)$ & $600(43 \%)$ & \\
\hline \multicolumn{5}{|l|}{ Comorbid conditions (yes) } \\
\hline Heart disease & $176(22 \%)$ & $53(20 \%)$ & $234(17 \%)$ & 0.01 \\
\hline Stroke & $29(4 \%)$ & $13(5 \%)$ & $24(2 \%)$ & 0.01 \\
\hline Hypertension & $267(34 \%)$ & $94(35 \%)$ & $502(36 \%)$ & 0.57 \\
\hline Asthma/COPD & $104(13 \%)$ & $40(15 \%)$ & $123(9 \%)$ & 0.001 \\
\hline Diabetes & $128(16 \%)$ & $52(19 \%)$ & $176(13 \%)$ & 0.004 \\
\hline Stomach disease & $15(2 \%)$ & $6(2 \%)$ & $20(1 \%)$ & 0.54 \\
\hline Kidney disease & $41(5 \%)$ & $19(7 \%)$ & $40(3 \%)$ & 0.001 \\
\hline Liver disease & $34(4 \%)$ & $19(7 \%)$ & $25(2 \%)$ & $<0.001$ \\
\hline Thyroid disease & $49(6 \%)$ & $12(5 \%)$ & $56(4 \%)$ & 0.07 \\
\hline Osteoarthritis & $223(28 \%)$ & $72(27 \%)$ & $343(25 \%)$ & 0.18 \\
\hline Rheumatoid arthritis & $68(9 \%)$ & $16(6 \%)$ & $80(6 \%)$ & 0.03 \\
\hline Back pain & $223(28 \%)$ & $66(25 \%)$ & $378(27 \%)$ & 0.50 \\
\hline BMI & $26.6(4.8)$ & $26.5(3.8)$ & $26.8(4.1)$ & 0.41 \\
\hline \multicolumn{5}{|l|}{ Behavior/well-being } \\
\hline Anxiety $(0-21)$ & $4.9(4.0)$ & $5.1(3.8)$ & $4.4(3.7)$ & $<0.001$ \\
\hline Depressive symptoms $(0-21)$ & $5.3(4.2)$ & $4.6(3.7)$ & $3.8(3.3)$ & $<0.001$ \\
\hline
\end{tabular}


Table 1 (continued)

\begin{tabular}{|c|c|c|c|c|}
\hline & One wave $N=891$ & Two waves $N=281$ & Full response (three waves) $N=1453$ & $p$ value \\
\hline Pain $(0-100)$ & $18.1(25.2)$ & $18.0(25.1)$ & $15.2(23.6)$ & 0.01 \\
\hline Insomnia $(0-100)$ & $21.9(29.8)$ & $23.1(29.1)$ & $19.9(27.6)$ & 0.11 \\
\hline Social functioning $(0-100)$ & $85.3(23.9)$ & $84.0(23.9)$ & $87.6(21.2)$ & 0.01 \\
\hline Emotional functioning $(0-100)$ & $84.1(20.8)$ & $83.5(20.1)$ & $87.4(18.2)$ & $<0.001$ \\
\hline Cognitive functioning $(0-100)$ & $84.1(20.5)$ & $82.7(22.2)$ & $85.8(19.9)$ & 0.03 \\
\hline Global quality of life $(0-100)$ & $73.4(21.1)$ & $75.1(18.1)$ & $79.7(17.6)$ & $<0.001$ \\
\hline \multicolumn{5}{|l|}{ Functional status } \\
\hline Physical functioning $(0-100)$ & $75.1(22.8)$ & $78.0(20.1)$ & $83.1(18.7)$ & $<0.001$ \\
\hline Role functioning (0-100) & $74.9(29.9)$ & $76.2(28.6)$ & $83.1(25.3)$ & $<0.001$ \\
\hline Occupation & & & & $<0.001$ \\
\hline Yes & $82(10 \%)$ & $51(18 \%)$ & $280(19 \%)$ & \\
\hline Moderate or vigorous physical activity (h/week) & $9.1(8.9)$ & $10.2(8.4)$ & $12.3(9.0)$ & $<0.001$ \\
\hline Meeting physical activity guidelines & & & & $<0.001$ \\
\hline Yes & $664(75 \%)$ & $239(86 \%)$ & $1314(91 \%)$ & \\
\hline Mean cancer-related fatigue & $21.8(7.6)$ & $21.7(7.0)$ & $19.9(6.4)$ & $<0.001$ \\
\hline Cancer-related fatigue & & & & $<0.001$ \\
\hline Yes & $352(43 \%)$ & $127(46 \%)$ & $485(34 \%)$ & \\
\hline
\end{tabular}

$(17 \%)$ and role functioning $(15 \%)$. The total model explained $63 \%$ of the variance in CRF levels.

\section{Discussion}

This study examined the course of CRF over time and its correlates among CRC survivors using a model adapted from a previously proposed conceptual framework. According to our results, multiple factors were associated with CRF over time: sociodemographic (sex, age, partner, educational level), clinical (chemotherapy, comorbid conditions, BMI), behavior/ well-being (insomnia, pain, anxiety, depression, social, cognitive, and emotional functioning), and functional status (physical and role functioning, being employed, physical activity). The total model explained $63 \%$ of the variance in CRF. The differences in CRF levels could, for a large part, be attributed to differences in behavior/well-being and functional status and, to a lesser extent, sociodemographic and clinical characteristics. The CRF levels were relatively stable over time, although significant within-person effects were found for behavior/well-being and functional status variables, which could be due to disease progression. All demographic characteristics were associated with levels of CRF, which is in accordance with the current literature and identifies groups at high risk for CRF. Younger cancer survivors, females, and lower educated survivors were more likely to feel fatigued [20-22]. Survivors with a partner were less likely to be fatigued, indicating the emotionally and instrumental supportive role of social support in addressing CRF [23].
Several studies among short-term cancer survivors found strong associations between clinical characteristics and levels of CRF [20, 24]. Our study among longer-term cancer survivors did not find significant temporal associations between disease stage, type of primary treatment, time since diagnosis, and levels of CRF, indicating that these factors become less important over time. Nevertheless, we found that survivors with two or more comorbid conditions were more likely to feel fatigued than those with either only one or without comorbidity. Similarly, other studies have reported that increasing numbers of comorbid conditions were associated with CRF [25]. We previously found that comorbidity explained a greater proportion of the variance in CRF scores among cancer survivors than clinical or sociodemographic variables [26].

Our results show that behavioral factors and well-being are significantly associated with CRF levels over time, which is consistent with previous literature demonstrating significant influences of physical distress factors like pain and insomnia and psychological distress factors like depression and anxiety on CRF [27]. It may be that these symptoms arise through a common pathway as previous research on inflammation and CRF suggests that tumors and the treatments used to treat them activate proinflammatory cytokines, leading to CRF and other symptoms [28].

Several previously conducted studies found significant associations between physical fitness and physical activity and levels of CRF among cancer survivors [27], which is in accordance with our finding of a negative association between functional status and CRF. Physical activity can counteract physical deconditioning and directly influence levels of CRF, or it 


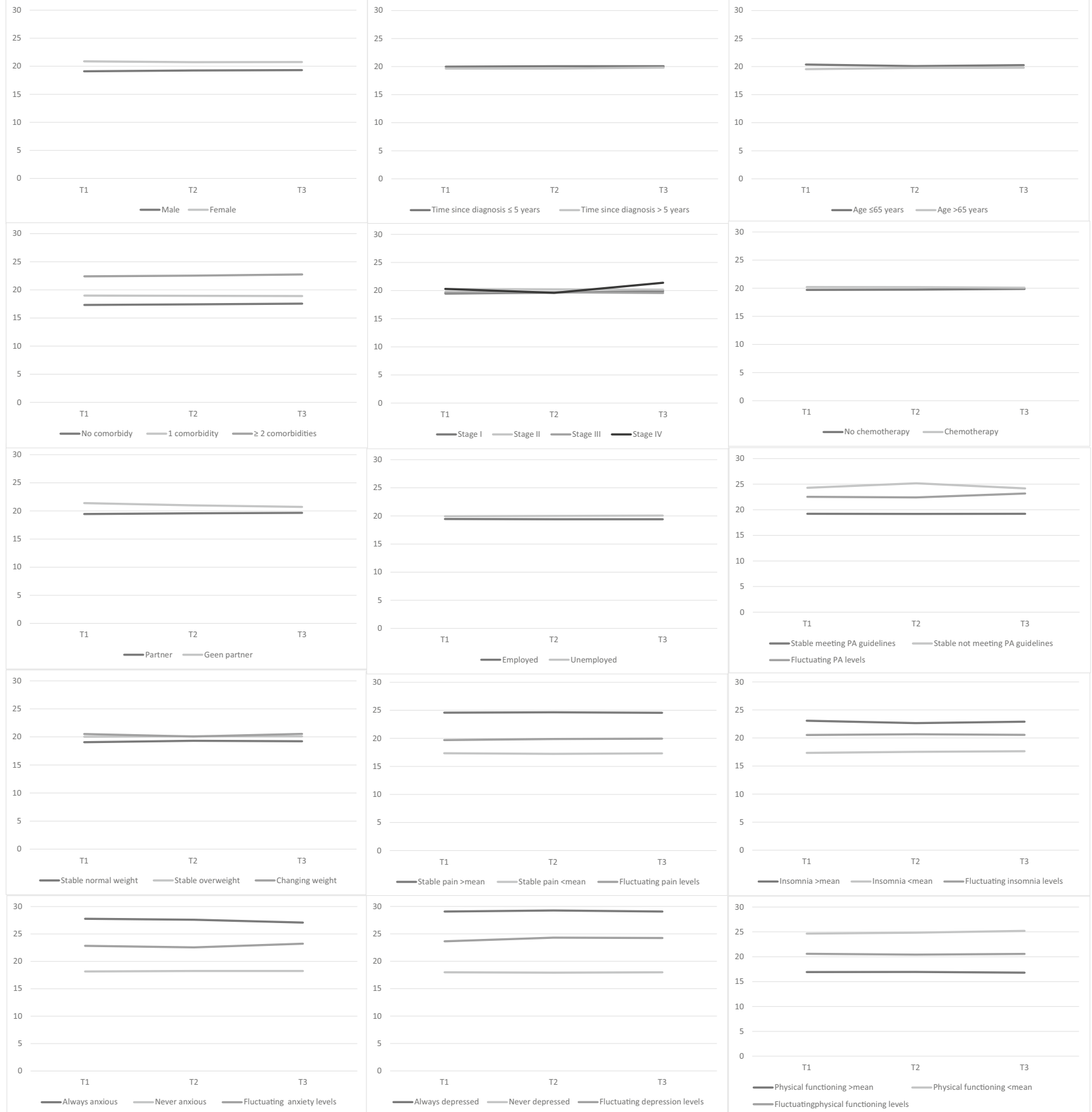

Fig. 3 Longitudinal changes in CRF over time for different correlates. Vertical axis represents CRF total scores (0-50); horizontal axis represents time points (T1-T3)

can reduce CRF indirectly by its beneficial effects on mood, immune functioning, or sleep [27].

Most sociodemographic characteristics remain stable over time. On the other hand, behavioral/well-being factors and functional status may be subject to change. Although the CRF levels remained relatively stable over time in our study, we did find within-subject effects for all behavioral/well-being and functional status factors. This indicates that levels of CRF can change by influencing these factors by means of interventions. Some interventions have shown efficacy in reducing CRF. Meta-analyses showed that psychosocial interventions (cognitive behavioral therapy, supportive-expressive therapy, education/counseling, behavioral/relaxation therapy) had a small to moderate effect on CRF [29-31], exercise interventions had a near moderate effect in reducing CRF [29, 31-36], and pharmacological interventions with methylphenidate, a sympathomimetic psychostimulant, was more effective in reducing CRF than a placebo [37]. Nevertheless, it remains 
Table 2 Adjusted linear mixed models estimating the individual associations between each independent variable and CRF over time

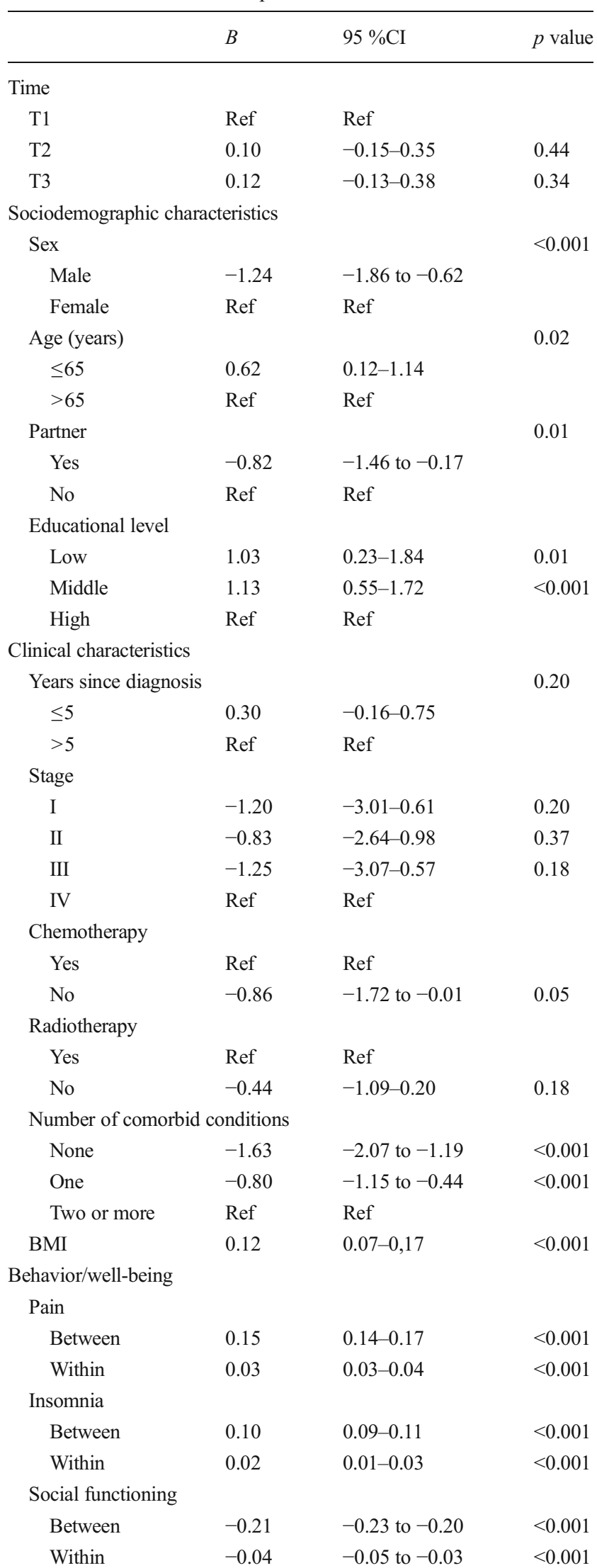

Table 2 (continued)

\begin{tabular}{|c|c|c|c|}
\hline & $B$ & $95 \% \mathrm{CI}$ & $p$ value \\
\hline \multicolumn{4}{|c|}{ Emotional functioning } \\
\hline Between & -0.23 & -0.24 to -0.21 & $<0.001$ \\
\hline Within & -0.07 & -0.08 to -0.06 & $<0.001$ \\
\hline \multicolumn{4}{|c|}{ Cognitive functioning } \\
\hline Between & -0.22 & -0.23 to -0.21 & $<0.001$ \\
\hline Within & -0.06 & $-0.07-0.05$ & $<0.001$ \\
\hline \multicolumn{4}{|c|}{ Global quality of life } \\
\hline Between & -0.27 & -0.28 to -0.25 & $<0.001$ \\
\hline Within & -0.07 & -0.08 to -0.06 & $<0.001$ \\
\hline \multicolumn{4}{|l|}{ Anxiety } \\
\hline Between & 1.04 & $0.97-1.12$ & $<0.001$ \\
\hline Within & 0.50 & $0.43-0.57$ & $<0.001$ \\
\hline \multicolumn{4}{|c|}{ Depressive symptoms } \\
\hline Between & 1.31 & $1.24-1.38$ & $<0.001$ \\
\hline Within & 0.60 & $0.54-0.67$ & $<0.001$ \\
\hline \multicolumn{4}{|c|}{ Functional status } \\
\hline \multicolumn{4}{|c|}{ Physical functioning } \\
\hline Between & -0.21 & -0.23 to -0.20 & $<0.001$ \\
\hline Within & -0.08 & -0.09 to -0.06 & $<0.001$ \\
\hline \multicolumn{4}{|c|}{ Role functioning } \\
\hline Between & -0.17 & -0.18 to -0.16 & $<0.001$ \\
\hline Within & -0.05 & -0.05 to -0.04 & $<0.001$ \\
\hline \multicolumn{4}{|l|}{ Occupation } \\
\hline Yes & Ref & Ref & \\
\hline No & 0.64 & $-0.21-1.51$ & 0.14 \\
\hline \multicolumn{4}{|c|}{ Moderate to vigorous physical activity level } \\
\hline Between & -0.14 & -0.18 to -0.10 & $<0.001$ \\
\hline Within & -0.03 & -0.05 to -0.01 & $<0.01$ \\
\hline
\end{tabular}

unclear which intervention (or combination of interventions) is best for each individual. A factor that makes the treatment of CRF complicated is that other symptoms such as pain, insomnia, and psychological distress frequently co-occur with CRF, as our results also indicate. The exact mechanisms of this cooccurrence are unclear: are all symptoms caused by cancer and its treatment? Are high pain levels causing high CRF levels or the other way around? And, which symptom(s) do we need to treat? It can be hypothesized that survivors with high levels of CRF and psychological distress will benefit most from a psychosocial intervention, while survivors with lower physical fitness will benefit from an exercise intervention. To personalize treatment of CRF, it may be an option for future studies to take a network approach. Instead of interpreting symptoms as a function of a set of underlying/latent factors, the network approach conceptualizes symptoms as mutually interacting, often reciprocally reinforcing elements of a complex network. Thus, rather than interpreting symptoms as measurements of a latent variable, symptoms are viewed as part of a causal system [38]. 
These causal systems can be different for each individual depending on centrality (which symptom is most important in one's network), connectivity (which symptoms are connected and how well are they connected), and distance (how long does it take for one symptom to influence another symptom). By studying the network of an individual cancer survivor, it becomes possible to target interventions at particular part of a person's network (e.g., the central symptom such as CRF) [39].

Some limitations of the present study should be mentioned. First, although information was present regarding demographic and clinical characteristics of the respondents and non-respondents, it remains unknown why non-respondents declined to participate. In addition, the differences found between respondents and non-respondents limit the generalizability of our results. Second, although our study has a longitudinal study design, it is still not possible to determine causality among the study variables, since the associations could also be influenced by variables that were not measured in the study. Further, we used the EORTC QLQ-C30 to assess different aspects of well-being; however, it could be argued that some constructs (e.g., cognitive functioning) could better be assessed with more specific questionnaires or even objective measures. Because all participants were CRC survivors, we can only generalize our results to this group of survivors. Last, we assessed an adapted model, as we do not have biological data. Nevertheless, this large population-based longitudinal study with good response rates provides a comprehensive view on several factors related to levels of CRF over time.

To conclude, this study showed that sociodemograpic and clinical factors were associated with CRF levels over time among CRC survivors; however, well-being, cancer-related symptoms, and functional status explained a larger part of the variance in levels of CRF.

Conflict of interest None of the authors has a conflict of interest.

Funding The present research is supported in part by a Veni grant (\#451-10-041) from the Netherlands Organization for Scientific Research (The Hague, The Netherlands) awarded to Floortje Mols, a Cancer Research Award from the Dutch Cancer Society (\#UVT-20094349) to Lonneke van de Poll-Franse, and a Social Psychology Fellowship from the Dutch Cancer Society to Melissa Thong (\#UVT2011-4960). Data collection for this study was funded by the Comprehensive Cancer Centre Netherlands, Eindhoven, The Netherlands, the Center of Research on Psychology in Somatic diseases (CoRPS), Tilburg University, The Netherlands, and an investment subsidy (\#480-08-009) of the Netherlands Organization for Scientific Research, The Hague, The Netherlands. The funding sources were involved neither in the collection, interpretation, and analysis of the data, nor in the decision for the writing and submission of this report for publication. The authors have full control over all primary data.

Open Access This article is distributed under the terms of the Creative Commons Attribution-NonCommercial 4.0 International License (http:// creativecommons.org/licenses/by-nc/4.0/), which permits any noncommercial use, distribution, and reproduction in any medium, provided you give appropriate credit to the original author(s) and the source, provide a link to the Creative Commons license, and indicate if changes were made.

\section{References}

1. Siegel R, Desantis C, Jemal A (2014) Colorectal cancer statistics, 2014. CA Cancer J Clin 64:104-117

2. Meulepas JM, Kiemeney LALM, Benraadt J (2011) Cancer in The Netherlands until 2020. Trends and prognoses (Kanker in Nederland Tot 2020: Trends En Prognoses). Kanker in Nederland Tot 2020: Trends En PrognosesSignaleringscommissie Kanker van KWF Kankerbestrijding, Vereniging van Integrale Kankercentra, Amsterdam, the Netherlands, 39

3. Cijfers over kanker. 2014. Available: http://www.cijfersoverkanker. nl. Accessed 19 December 2014

4. Berger AM, Abernethy AP, Atkinson A et al (2010) Cancer-related fatigue. J Natl Compr Cancer Netw 8:904-931

5. Servaes P, Verhagen C, Bleijenberg G (2002) Fatigue in cancer patients during and after treatment: prevalence, correlates and interventions. Eur J Cancer 38:27-43

6. Thong MSY, Mols F, Wang XS et al (2013) Quantifying fatigue in (long-term) colorectal cancer survivors: a study from the population-based patient reported outcomes following initial treatment and long term evaluation of survivorship registry. Eur $\mathbf{J}$ Cancer 49:1957-1966

7. Hofman M, Ryan JL, Figueroa-Moseley CD et al (2007) Cancerrelated fatigue: the scale of the problem. Oncologist 12(Suppl 1):4 10

8. Barsevick AM, Irwin MR, Hinds P et al (2013) Recommendations for high-priority research on cancer-related fatigue in children and adults. J Natl Cancer Inst 105:1432-1440

9. van de Poll-Franse LV, Horevoorts N, Eenbergen MV et al (2011) The patient reported outcomes following initial treatment and long term evaluation of survivorship registry: scope, rationale and design of an infrastructure for the study of physical and psychosocial outcomes in cancer survivorship cohorts. Eur J Cancer 47:2188-94

10. Michielsen HJ, Drent M, Peros-Golubicic T, De Vries J (2006) Fatigue is associated with quality of life in sarcoidosis patients. Chest 130:989-994

11. Michielsen HJ, De Vries J, Van Heck GL (2003) Psychometric qualities of a brief self-rated fatigue measure: the fatigue assessment scale. J Psychosom Res 54:345-352

12. Sangha O, Stucki G, Liang MH et al (2003) The self-Administered Comorbidity Questionnaire: a new method to assess comorbidity for clinical and health services research. Arthritis Rheum 49:156163

13. Aaronson NK, Ahmedzai S, Bergman B et al (1993) The European Organization for Research and Treatment of Cancer QLQ-C30: a quality-of-life instrument for use in international clinical trials in oncology. J Natl Cancer Inst 85:365-376

14. Zigmond AS, Snaith RP (1983) The hospital anxiety and depression scale. Acta Psychiatr Scand 67:361-370

15. Pols MA, Peeters PH, Ocke MC et al (1997) Estimation of reproducibility and relative validity of the questions included in the EPIC Physical Activity Questionnaire. Int J Epidemiol 26(Suppl 1): S181-189

16. Ainsworth BE, Haskell WL, Leon AS et al (1993) Compendium of physical activities: classification of energy costs of human physical activities. Med Sci Sports Exerc 25:71-80

17. Ainsworth BE, Haskell WL, Whitt MC et al (2000) Compendium of physical activities: an update of activity codes and MET intensities. Med Sci Sports Exerc 32:S498-504 
18. Hedeker DR, Gibbons RD (2006) Longitudinal data analysis. Chapter 6. Wiley, Hoboken

19. Cohen J, Cohen P, West SG, Aiken LS (2003) Applied multiple regression/correlation analysis for the behavioral sciences. Chapter 7. Erlbaum, Mahwah

20. Jansen L, Herrmann A, Stegmaier C et al (2011) Health-related quality of life during the 10 years after diagnosis of colorectal cancer: a population-based study. J Clin Oncol 29:3263-3269

21. Baldwin CM, Grant M, Wendel C et al (2009) Gender differences in sleep disruption and fatigue on quality of life among persons with ostomies. J Clin Sleep Med 5:335-343

22. Wu H-S, Harden JK (2015) Symptom burden and quality of life in survivorship: a review of the literature. Cancer Nurs 38:E29-54

23. Soares A, Biasoli I, Scheliga A et al (2013) Association of social network and social support with health-related quality of life and fatigue in long-term survivors of Hodgkin lymphoma. Support Care Cancer 21:2153-2159

24. Goedendorp MM, Andrykowski MA, Donovan KA et al (2012) Prolonged impact of chemotherapy on fatigue in breast cancer survivors: a longitudinal comparison with radiotherapy-treated breast cancer survivors and noncancer controls. Cancer 118:3833-3841

25. Barbera L, Seow H, Howell D et al (2010) Symptom burden and performance status in a population-based cohort of ambulatory cancer patients. Cancer 116:5767-5776

26. Vissers PAJ, Thong MS, Pouwer F et al (2013) The impact of comorbidity on Health-Related Quality of Life among cancer survivors: analyses of data from the PROFILES registry. J Cancer Surviv 7:607-13

27. Seo Y, Oh H, Seo W (2010) Causal relationships among factors associated with cancer-related fatigue. Eur J Oncol Nurs 14:380 386

28. Bower JE, Lamkin DM (2013) Inflammation and cancer-related fatigue: mechanisms, contributing factors, and treatment implications. Brain Behav Immun 30(Suppl):S48-57

29. Duijts SF, Faber MM, Oldenburg HS et al (2011) Effectiveness of behavioral techniques and physical exercise on psychosocial functioning and health-related quality of life in breast cancer patients and survivors-a meta-analysis. Psychooncology 20: 115-126

30. Jacobsen PB, Donovan KA, Vadaparampil ST, Small BJ (2007) Systematic review and meta-analysis of psychological and activity-based interventions for cancer-related fatigue. Health Psychol 26:660-667

31. Kangas M, Bovbjerg DH, Montgomery GH (2008) Cancer-related fatigue: a systematic and meta-analytic review of nonpharmacological therapies for cancer patients. Psychol Bull 134: 700-741

32. Arnold M, Taylor NF (2010) Does exercise reduce cancer-related fatigue in hospitalised oncology patients? A systematic review. Onkologie 33:625-630

33. Brown JC, Huedo-Medina TB, Pescatello LS et al (2011) Efficacy of exercise interventions in modulating cancer-related fatigue among adult cancer survivors: a meta-analysis. Cancer Epidemiol Biomarkers Prev 20:123-133

34. McMillan EM, Newhouse IJ (2011) Exercise is an effective treatment modality for reducing cancer-related fatigue and improving physical capacity in cancer patients and survivors: a meta-analysis. Appl Physiol Nutr Metab 36:892-903

35. Cramp F, Daniel J (2008) Exercise for the management of cancerrelated fatigue in adults. The Cochrane database of systematic reviews. CD006145

36. Velthuis MJ, Agasi-Idenburg SC, Aufdemkampe G, Wittink HM (2010) The effect of physical exercise on cancer-related fatigue during cancer treatment: a meta-analysis of randomised controlled trials. Clin Oncol (R Coll Radiol) 22:208-221

37. Minton O, Richardson A, Sharpe M et al (2008) A systematic review and meta-analysis of the pharmacological treatment of cancer-related fatigue. J Natl Cancer Inst 100:1155-1166

38. Borsboom D (2008) Psychometric perspectives on diagnostic systems. J Clin Psychol 64:1089-1108

39. Borsboom D, Cramer AO (2013) Network analysis: an integrative approach to the structure of psychopathology. Annu Rev Clin Psychol 9:91-121 